\section{Abstractions}

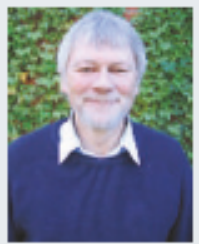

LAST AUTHOR

Understanding the way

people police use of

shared resources - such

as fishing stocks or forests

- could offer clues to

rescuing these resources

when they come under

threat. Manfred Milinski, an evolutionary ecologist at the Max Planck Institute of Limnology in Plön, Germany, teamed up with economist Bettina Rockenbach of the University of Erfurt to determine ways to encourage sharing. They used an interactive monetary game to see how players reward or punish each other, depending on their behaviour (see page 718 ).

How did an economist and an evolutionary biologist come together for this work? Using iterative gamesin which subjects contribute to a common pool of money, empirical economists recently found that exacting punishment - by forcing noncooperators to contribute more during the course of the game - enhanced cooperation. Meanwhile, evolutionary biologists found that building a solid reputation as a giver encouraged contributions from all. Bettina and Imet at a workshop and decided to join forces.

You thought that the subjects would give up punishing behaviour. Was that the case? In successive rounds of the experiment, we expected people to give up punishing behaviour because punishment has higher costs than withholding support from members who had accumulated a bad social reputation. We found that the subjects chose not to impose punishment that often, but they didn't want to get rid of it completely. We also found that combining punishment and reputation-building as a giver boosted cooperative efficiency.

It seems from your work that humans have a particular disdain for 'f ree riders'. Why? As soon as afree rider enters the game, the others give up contributing to the public pool. The introduction of one free rider causes breakd own of cooperation. People have strong emotions against free riders, who are often seen as parasites.

Are humans gluttons for punishment? When people were given a choice, they preferred to have both reputation and punishment available as tools to promote cooperation. Even though they used punishment at alow level, they preferred having a lastresort. If reputation doesn't help, punishmentprovides the teeth necessary to discipline the worst guys.

What are the implications of your study? Introducing publicity mighthelp cure a 'public goods' dilemma. If people are seen overusing apublic resource, they might stop.
MAKING THE PAPER

\section{Yannick De Wilde}

\section{How to build a nanoscopic version of a night-vision camera.}

The idea of thermal imaging is not new. Nightvision cameras using this technique generate their images by detecting the infrared radiation given off by objects. On page 740, Yannick De Wilde takes this concept a step further in the shape of an atomic-scale microscope that works by detecting the infrared radiation given off naturally by surfaces.

De Wilde built his first microscope as a postdoc at Argonne National Laboratory in Illinois. The instrument was a scanning tunnelling microscope (STM), which detects the position of electrons using a slight electric current between the tip of a probe and the surface of the material being scanned. This allows areas containing a lot of electrons to be identified, information that can be used to infer the position of atoms and molecules on the surface. But STMs do not work with materials that cannot conduct electricity.

In 2000, De Wilde took up a post at the optics lab at the City of Paris Industrial Physics and Chemistry Higher Educational Institution in France. There he began work on a variation of the STM in which the probe shone infrared light on the surface being scanned and detected the light reflected back. This microscope, one of two such instruments in the world at the time, generated optical images at unprecedented resolution - as good as 50 nanometres or less - and worked with most materials, regardless of whether or not they were electrical conductors.

In 2002, De Wilde decided to push the envelope further. He set about trying to build a version of the new microscope that would not emit light but would generate images using the infrared light given offby materials. "Instead of determining the local density of electrons in a sample, the microscope would look at the

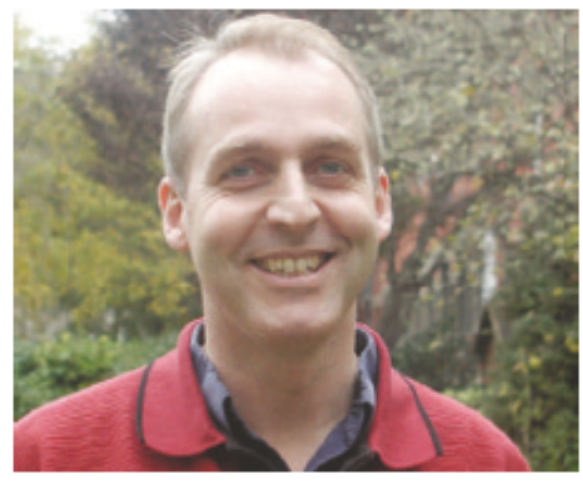

density of photons, ${ }^{n}$ De Wilde says. It seemed like a good idea, although some researchers in the field thought it would prove difficult to implement. ${ }^{\alpha}$ They thought there would not be enough energy to be able to measure it with the tiny probe," says De Wilde. After all, detecting a signal using infrared light had been tricky enough. If he removed the light source altogether the signal would become even weaker. "We were dealing with signals three orders of magnitude weaker than with the infrared microscope," says De Wilde.

But a chance meeting with several colleagues pointed him to the way forward. For one thing, his colleagues had shown that some materials have high electromagnetic energy confined to their surface, which can be further enhanced by raising the temperature. So De Wilde and a $\mathrm{PhD}$ student in his lab, Florian Formanek, tried heating their sample to generate a signal strong enough to be detected by the microscope. But the heat made the probe unstable. So they redesigned the probe to withstand the heat. It took two years of trial and error before they got an image of gold nanostructures on a surface of silicon carbide, showing that their light-free microscope worked.

But De Wilde has not finished designing new microscopes. Heplans to tweak his instrument further to make it even more sensitive. His goal is an instrument that can detect not only the position of photons, but also the spectrum of their energies, revealing even more information about the physical properties of substances.

\title{
KEY EXPERIMENT
}

\section{A landmark experiment from} 1956 is followed up on page 733 by physicist Immanuel Bloch and his colleagues at the Johannes Gutenberg University in Mainz, Germany. They have investigated aspects of the Hanbury Brown-Twiss effect, which deals with the quantum effects of particle interference. In their original work, Robert Hanbury Brown and Richard Twiss used two detectors to pick up photons from a single light source - in their case a star. They discovered that the detected photons 'bunched' in other words if a photon was detected at one of the detectors, it was highly likely that a photon would be detected at the other detector at the same time. This effect is seen for all bosons - particles such as photons or certain neutral atoms. But when it comes to fermions, such as quarks or fermionic atoms, the opposite should be true - a phenomenon known as antibunching.
Antibunching was first seen with electrons in semiconductors. In the present experiment, Bloch and his team have detected it for the first time in neutral fermionic atoms - which has enabled them to reveal properties of cold quantum gases released from optical lattices. In future experiments, they hope to use the technique to examine the atomic mechanisms of hightemperature superconductivity in such gases. 